Results For most depressed outpatients, sertraline, escitalopram or bupropion are reasonable first choices. If there is no response, the prescriber has many choices for the second trial in this algorithm because there is no clear preference based on evidence, and there are many individual patient considerations and variations in patient preference to take into account. The prescriber and patient may decide to either switch (to one of the above options not previously tried, or to venlafaxine, or to a nutraceutical antidepressant such as St. John's Wort or S-adenosylmethionine, or to transcranial magnetic stimulation), or to augment (with nutrients including l-methylfolate, or second-generation antipsychotics, or mirtazapine, or lithium or triiodothyronine). If there is no response to the second medication trial, the patient is considered to have a relatively medication-resistant depression. More recommendations follow. Comorbidities such as chronic pain, obsessivecompulsive disorder, attention-deficit hyperactivity disorder and posttraumatic stress disorder are considered.

Conclusions Utilization of this consultative tool for picking medication treatment for depression could help minimize unproductive variation in clinical care and improve clinical outcomes and produce remissions in shorter times and with fewer medication changes than with treatment as usual. Also, the algorithm encourages more cost-effective practice when generic options are recommended over expensive, brand-name products (when there is no apparent disadvantage in outcome or safety). Clinicians often overlook the large role their beliefs play in medication selection, ignoring placebo effects that contribute to their experience-based biases. These online tools provide evidence-supported ways of thinking that are available rapidly at the point of care in time to influence decisionmaking.

\section{VALIDATION OF CROWDSOURCING FOR CITATION SCREENING IN SYSTEMATIC REVIEWS}

\footnotetext{
${ }^{1,2}$ Nassr Nama, ${ }^{3}$ Margaret Sampson, ${ }^{4,5}$ Nick Barrowman, ${ }^{3}$ Katie O'Hearn, ${ }^{6}$ Ryan Sandarage, ${ }^{4,3}$ Kusum Menon, ${ }^{4,3}$ Gail Macartney, ${ }^{4,3}$ Kimmo Murto, ${ }^{4,3}$ Jean-Philippe Vaccani, ${ }^{4,3}$ Sherri Katz, ${ }^{4,3}$ Roger Zemek, ${ }^{4,3}$ Ahmed Nasr, ${ }^{4,3}$ James Dayre McNally. ${ }^{1}$ University of British Columbia, Faculty of Medicine, Vancouver, Canada; ${ }^{2} B C$ Children's Hospital, Vancouver, Canada; ${ }^{3}$ Children's Hospital of Eastern Ontario, Ottawa, Canada; ${ }^{4}$ University of Ottawa, Faculty of Medicine, Ottawa, Canada; ${ }^{5}$ Clinical Research Unit, CHEO Research Institute, Ottawa, Canada; ${ }^{6}$ University of British Columbia, Faculty of Medicine, Vancouver, Canada
}

\subsection{6/bmjebm-2019-EBMLive.49}

Objectives Systematic reviews (SRs) are often cited as the highest level of evidence available as they involve the identification and synthesis of published studies on a topic. Unfortunately, it is increasingly challenging for small teams to complete SR procedures in a reasonable time period, given the exponential rise in the volume of primary literature. Crowdsourcing has been postulated as a potential solution. The feasibility objective of this study was to determine whether an online crowd would be willing to perform and complete abstract and full text screening. The validation objective was to assess the quality of the crowd's work, including retention of eligible citations (sensitivity) and work performed for the investigative team, defined as the percentage of citations excluded by the crowd.

Method We performed a prospective study evaluating the feasibility and validity of crowdsourcing essential components of an SR, including abstract screening, document retrieval, and full text assessment. Using the CrowdScreenSR citation screening software, 2323 articles from 6 SRs were available to an online crowd. Citations excluded by less than or equal to $75 \%$ of the crowd were moved forward for full text assessment. For the validation component, performance of the crowd was compared with citation review through the accepted, gold standard, trained expert approach.

Results Of 312 potential crowd members, 117 (37.5\%) commenced abstract screening and $71(22.8 \%)$ completed the minimum requirement of 50 citation assessments. The majority of participants were students $(192 / 312,61.5 \%)$. The crowd screened 16,988 abstracts (median: 8 per citation; IQR 7-8), and all citations achieved the minimum of 4 assessments after a median of 42 days (IQR 26-67). Crowd members retrieved $83.5 \%(774 / 927)$ of the articles that progressed to the full text phase. A total of 7604 full text assessments were completed (median: 7 per citation; IQR 3-11). Citations from all but 1 review achieved the minimum of 4 assessments after a median of 36 days (IQR 24-70). When complete crowd member agreement at both levels was required for exclusion, sensitivity was $100 \%(95 \% \mathrm{CI} 97.9-100)$ and work performed was $68.3 \%$ (95\%CI 66.4-70.1). Using the predefined alternative $75 \%$ exclusion threshold, sensitivity remained $100 \%$ and work performed increased to $72.9 \%$ (95\%CI 71.0-74.6; $P<.001)$.

Conclusions Crowdsourcing of citation screening for SRs is feasible and has reasonable sensitivity and specificity. By expediting the screening process, crowdsourcing could permit the investigative team to focus on more complex SR tasks. This requires a user-friendly online platform that allows research teams to crowdsource their reviews. Future directions should focus on assessing the application of this methodology to real life projects and determine its potential for rapid completion of systematic reviews.

\section{INVESTIGATOR'S BROCHURES: DO THEY ADHERE TO BASIC PRINCIPLES OF EVIDENCE SYNTHESIS METHODS?}

${ }^{1}$ Johannes Schwietering, ${ }^{2,3}$ Merlin Bittlinger, ${ }^{1-3}$ Daniel Strech. ${ }^{1}$ Hannover Medical School, Hannover, Germany; ${ }^{2}$ Berlin Institute of Health, Berlin, Germany; ${ }^{3}$ Charité Universitätsmedizin Berlin, Berlin, Germany

\subsection{6/bmjebm-2019-EBMLive.50}

Objectives The purpose of investigator's brochures (IB) is to compile the relevant evidence in order to enable an informed risk-benefit assessment by different reviewers including the principal investigator, a research ethics committees (REC), regulatory authorities, or data safety monitoring boards. Although a vast literature exists on the methodology of evidence synthesis for systematic reviews and meta-analyses, there is almost no literature examining the role of evidence synthesis in IBs. The primary objective of this contribution is to examine the adherence of IBs to fundamental principles of knowledge synthesis. These principles include a systematic search strategy, an evaluation of the risk of bias of the included studies and a comprehensive data collection and transparent synthesis procedure that accounts for variation in information quality.

Method We systematically examined a random sample of 30 IBs of a large sample $(\mathrm{N}=109)$ from the application materials of industry-sponsored pharmaceutical clinical trials conducted between 2010-2016. IBs were obtained from three RECs of German university medical centers under data confidentiality agreements. Multiple independent examiners assessed the IBs to identify clinical trials reported in the IBs and 